\title{
Beyond two generations
}

\author{
Georgios Anagnostou, on behalf of the CMS Collaboration ${ }^{1, a}$ \\ ${ }^{1}$ Institute Of Nuclear and Particle Physics, NCSR Demokritos
}

\begin{abstract}
A review is presented of searches performed by CMS experiment for new particles produced in association with or decaying to top quarks, as well as heavy top partners. The analysis presented use data collected with CMS experiment during 2012, in proton-proton collisions at a centre-of-mass energy of $8 \mathrm{TeV}$.
\end{abstract}

\section{Introduction}

After the discovery of a SM-like Higgs boson $[1,2]$, an explanation for the scale of its mass became of great importance. New particles, such as heavy top partners, could improve our understanding of the problem, through cancelations in the radiative corrections of the Higgs boson mass.

Many searches have been performed by CMS experiment [3] for new particles produced in association with or decaying to top quarks, as well as heavy top partners [4]. In this high energy regime, the top quarks become boosted and their decay products are oftenly collimated. Sophisticated algorithms have been developed for tagging top quarks and estimating the mass of their hadronic decays [5-7]. These techniques allow the reconstruction of invariant mass observables in many final states, a powerful tool to discrimimate possible new resonances from their background processes. Mass reconstruction methods are also developed for leptonic final states including one or two invisible particles [8-10].

This report summarizes many of the searches performed by the CMS for heavy top partners as well as new particles produced in association with or decaying to top quarks [8-18]: top quarks associated with possible dark matter particles (section 2) and resonances decaying to top quarks (sections 3, 4, 5). Finally, searches for heavy top partners are presented in sections 6, 7 .

\section{Dark matter searches}

Theories that attempt to extend the Standard Model like supersymmetry or extra dimensions, predict the existence of neutral weakly interacting particles which escape detection in collider experiments. Final states with a single jet $[19,20]$ or photon $[21,22]$ associated with large missing energy have been searched by both ATLAS and CMS without any evidence for new physics. This result can be accomodated by models where the new particle converts a light quark to a top quark, predicting "monotop" candidates, that is an invisible particle in association with a top quark [23, 24]. The CMS search [11] is focused on the hadronic final state, in which top decays to a bottom quark and a $\mathrm{W}$

a e-mail: Georgios.Anagnostou@ cern.ch 

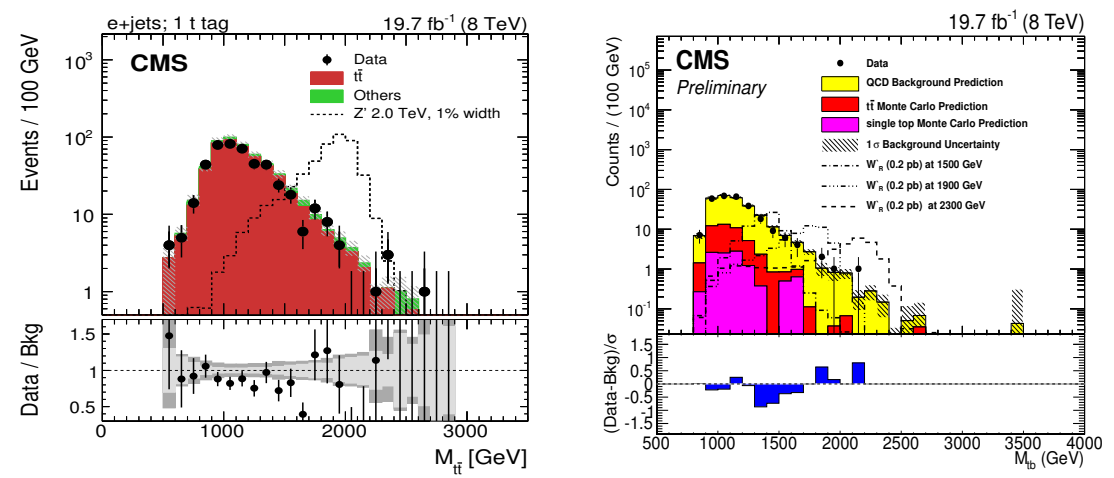

Figure 1. Invariant mass of the reconstructed $t \bar{t}$ pair in the electron+jet channel for events with $1 \mathrm{t}$-tagged jet (left) [13], and the reconstructed $\mathrm{M}_{\mathrm{tb}}$ invariant mass in the all-hadronic $\mathrm{W}^{\prime} \rightarrow \mathrm{tb}$ search (right) [15].

boson, which further decays into a pair of quarks. Events with large missing energy and three jets are selected and one of them must be identified as originating from a $b$ quark. The signal events as well as the number of multijet background events, are measured simulatenously using a likelihood approach. The observed lower limits for the invisible particle are $330 \mathrm{GeV}$ for a scalar and $650 \mathrm{GeV}$ for a vector particle respectively.

Another scenario predicts a new fermion interacting with quarks via a four-fermion contact interaction $[25,26]$. The exclusion limit for the scalar case is the least stringent among all types that have been searched. In addition, the interaction coupling strength is proportional to the mass of the quark, predicting better sensitivity in final states with third-generation quarks [27]. The CMS search is focused on the scalar case, in the final state originating from the semi-leptonic top pair decay. The selection requires events with a single isolated lepton, at least three jets of which one must be identified as originating from $b$ quark fragmentation and large missing transverse energy [12]. Further selection criteria are based on the transverse mass of the lepton and missing energy, as well as $\mathrm{M}_{\mathrm{T} 2}^{\mathrm{W}}$ variable [28], in order to suppress $\mathrm{W}+$ jets and top pair backgrounds. The upper limits on the $\mathrm{pp} \rightarrow \mathrm{t} \overline{\mathrm{t}}+\mathrm{X} \overline{\mathrm{X}}$ production cross section depend on the mass hypothesis of the DM particle: cross sections larger than 20 to $55 \mathrm{fb}$ are excluded for DM particles ranging from 1 to $1000 \mathrm{GeV}$.

\section{Resonances decaying to top pairs}

A resonance decaying to top pairs is predicted by many BSM theories like leptophobic $\mathrm{Z}^{\prime}$ [29], extended gauge theories [30] or extensions of the Randal-Sundrum model with Kaluza-Klein (KK) excitations [31]. The CMS search for resonances decaying to top quarks [13] is performed in final states with two, one or zero leptons refered to as dilepton, lepton+jet and all hadronic channels respectively.

The dilepton final states requires two non-isolated leptons (electrons or muons) of opposite charge, at least two jets, as well as large transverse missing energy. The final observable is a mass variable constructed from the vectorial sum of the above. The lepton+jet final state selects one electron or muon, at least two jets and large missing energy. The CMS top tagging algorithm is applied to identify the fully hadronic top decays merged into a single jet, using Cambridge-Aachen jets with a distance parameter of $\mathrm{R}=0.8$ (CA8 jets) [5, 6]. Events are categorized based on the lepton flavour and the number of CA8 t-tagged jets, from which no more than one is allowed to avoid overlap with the all- 


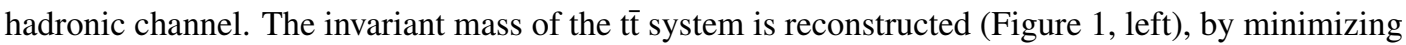
a $\chi^{2}$ function based on the masses of the possible $\mathrm{t} \overline{\mathrm{t}}$ candidates.

The all-hadronic final state requires a di-jet topology in which each jet is consistent with the decay of a top quark. The search is performed in two different $\mathrm{Z}^{\prime}$ mass regions: in the $\mathrm{M}_{\mathrm{Z}^{\prime}}$ region below $1 \mathrm{TeV}$ the Cambridge-Aachen (CA) jet reconstruction is applied with a distance parameter of $\mathrm{R}=1.5$ and HEPTopTagger algorithm [7], whereas in the high mass region the top jets are reconstructed using an $\mathrm{R}=0.8$ and CMS t-tagging algorithm $[5,6]$. In order to reduce the dominant multijet production, b-tagging is applied to the subjets of top, requiring one of them to originate from the fragmentation of $\mathrm{a} b$ quark. The combination of the above channels for resonances decaying to top pairs gives an exclusion range up to $2.4 \mathrm{TeV}$ for the narrow $\mathrm{Z}^{\prime}$ hypothesis. For wide resonances (width $10 \%$ of its mass) the exclusion range is up to $2.9 \mathrm{TeV}$. Randall-Sundrum KK gluons decaying to top pairs are excluded for masses below $2.8 \mathrm{TeV}$.

\section{Excited top quark}

The proposal that the top quark might have a composite structure [32], can be directly tested by searching for an excited top quark. The CMS search [8] adopts a model in which pair produced t* quark decays predominantly to a top quark and a gluon [33]. The analysis is targeting the lepton+jets final state ( $\mathrm{t}^{*} \mathrm{t}^{*} \rightarrow \operatorname{t\overline {t}gg} \rightarrow 1^{+} v_{1} \mathrm{~b} \overline{\mathrm{q}} \mathrm{q}^{\prime} \overline{\mathrm{b}} \mathrm{gg}$ ), requiring an isolated lepton and at least six jets, of which one must be combatible with originating from $b$ quark fragmentation. A kinematic fit is performed to final state objects in order to reconstruct $t^{*}$ candidates in each event. The fit is subject to the six mass constraints from excited top $\mathrm{t}^{*}$, top quark and $\mathrm{W}$ boson decays in both brances of the pair production. The analysis estimates a lower limit for $\mathrm{m}_{\mathrm{t}^{*}}$ of $803 \mathrm{GeV}$ for the combined electron and muon channels.

A search for pair produced resonances decaying to a top quark and another parton is also performed by CMS in the dilepton final state [9]. The experimental signature constists of two isolated leptons (electrons or muons), two jets coming from the decays of $b$ quarks and two jets originating from light flavour quarks or gluons. The interpretation is based on a spin-3/2 top quark excitation model, as in the lepton+jets final state [34]. An additional interpretation is also used, based on an R-parity violating MSSM model with the LSP bottom squark decaying into a top quark and a strange quark [35]. The invariant mass of the $t^{*}$ or $\tilde{b}$ resonance can be reconstructed: given the masses of the top quark and the $\mathrm{W}$ boson, the $\mathrm{t} \overline{\mathrm{t}}$ system can be solved analytically [36, 37]. The quadric equation can be solved for the two leptons and b-tagged jets combinations to form $t \bar{t}$ candidates which can further be combined with the selected light jets to form pairs of $t^{*}$ or $\tilde{b}$ candidates. The pair with the closest $\mathrm{M}_{\mathrm{t}, \mathrm{jet}}$ values is chosen and the average value of the pair is used as the final reconstructed mass. The observed upper limit for $\mathrm{t}^{*}$ and $\tilde{\mathrm{b}}$ resonances is $703 \mathrm{GeV}$ and $326 \mathrm{GeV}$ respectively.

\section{$5 \mathrm{t}+\mathrm{b}$ resonances}

A new massive charged gauge boson $\mathrm{W}^{\prime}$ is predicted by many extensions of the SM [38, 39] and has been searched in the lepton-neutrino, diboson and light quark final states [40-42]. The most important experimental constraints imposed by the leptonic final state, do not apply for the case of $\mathrm{W}^{\prime}$ bosons with purely right-handed couplings and a hypothetical right-handed neutrino mass larger than a few $\mathrm{GeV}$ [43]. CMS has performed further searches for $\mathrm{a} \mathrm{W}^{\prime+} \rightarrow \mathrm{t} \overline{\mathrm{b}}$ (or charge conjugate) in the lepton+jets and all-hadronic final state $[14,15]$.

The lepton+jets search [14] selects events with an isolated lepton (electron or muon), at least two jets of which one must originate from b-quark fragmentation. The topology $\mathrm{W}^{\prime} \rightarrow \mathrm{tb}, \mathrm{t} \rightarrow \mathrm{bW} \rightarrow \mathrm{bl} v$ is fully reconstructable: The $\mathrm{z}$ component of the neutrino momentum is calculated using the $\mathrm{W}$-boson 

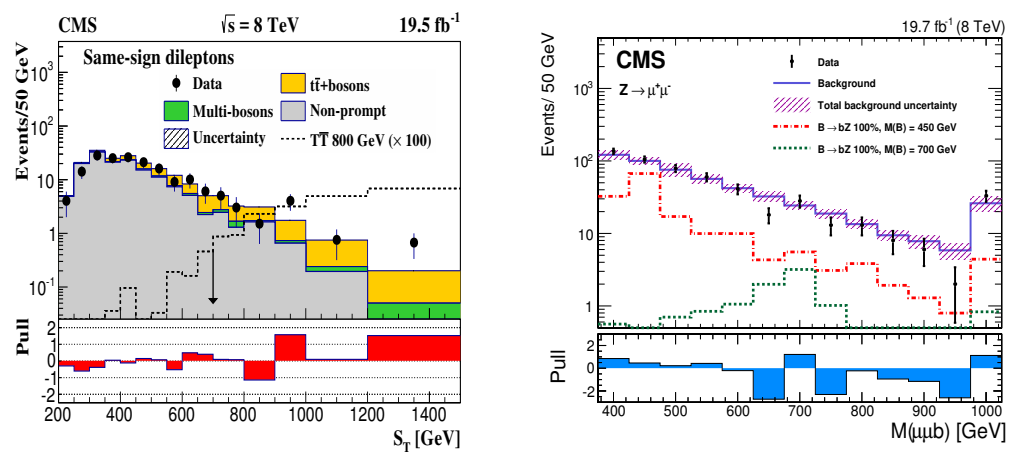

Figure 2. Observed and excpected $\mathrm{S}_{\mathrm{T}}$ distribution (same-sign dilepton sample) in the vector-like $\mathrm{T}$ quark search (left) [17], and the invariant mass of reconstructed B quark candidates in the opposite-sign dimuon channel (right) [18].

mass constraint. Each W-candidate is combined with all selected jets to form top-quark candidates. The candidate with invariant mass closest to top-quark mass is chosen and is further combined with the highest -in $\mathrm{P}_{\mathrm{T}^{-}}$of the remaining jets to form a $\mathrm{W}^{\prime}$ candidate. The observed limit for purely right-handed $\mathrm{W}^{\prime}$ bosons is $2.05 \mathrm{TeV}$. For a $\mathrm{W}^{\prime}$ boson with purely left-handed couplings including interefence effects, the observed limit is $1.84 \mathrm{TeV}$.

In the all-hadronic $\mathrm{W}^{\prime} \rightarrow \mathrm{t} \overline{\mathrm{b}}$ search [15], the Cambridge-Aachen algorithm is used for jet reconstruction (CA8 jets) and the CMS top-tagger for top jet identification. The selection requires a back-to-back dijet topology composed of a t-tagged jet and a low mass b-tagged jet which further allow the reconstruction of the $\mathrm{W}^{\prime}$ candidate mass (Figure 1, right). The production of a right-handed $\mathrm{W}^{\prime}$ boson in the all-hadronic search is excluded for masses up to $2.02 \mathrm{TeV}$. The combination of both lepton+jets and all-hadronic channels increase the exclusion range up to $2.15 \mathrm{TeV}$.

\section{Vector-like quarks}

Vector-like quarks are hypothetical new quarks for which both left and right-handed fields have the same transformation properties under the electroweak gauge group [45]. They do not need to receive their masses from Yuakawa couplings to Higgs doublet, as their bare mass terms are invariant under the symmetry of the theory. So these models are constistent with existing Higgs data, in contrast with models predicting extra quarks with chiral couplings such as fourth generation quarks [46].

The CMS search for top-quark partners with an exotic charge [16] is looking for pair produced new particles $\mathrm{T}_{5 / 3}$ with charge $5 \mathrm{e} / 3$ and decay via $\mathrm{T}_{5 / 3} \rightarrow \mathrm{tW}^{+}$, followed by $\mathrm{t} \rightarrow \mathrm{W}^{+} \mathrm{b}$ (and charge conjugates). The analysis is performed in the same-sign final state: it has less $t \bar{t}$ background but also instrumental effects from charge misidentification and fake leptons which are estimated from control regions. In addition to the CMS top-tagging, a W-tagging algorithm is applied to the CA8 jets, requiring exactly two subjets and their mass to be consistent with a $\mathrm{W}$ boson mass [44]. The selection requires two isolated same-sign leptons, large scalar sum of the $\mathrm{P}_{\mathrm{T}}$ of all leptons/jets and the number of CA8 subjets, leptons and anti-kt jets to be greater than six. The observed limit on the mass of $\mathrm{T}_{5 / 3}$ is $800 \mathrm{GeV}$.

CMS has performed an inclusive search for a vector-like T quark with charge $2 / 3$, which further decays to bW, $\mathrm{tZ}$ or $\mathrm{tH}$, in single-lepton and mulitlepton final states [17]. The single lepton channel 
requires at least four jets and uses a BDT to further separate T-quark signal from SM background. The multilepton channel requires at least two leptons and is further divided into four mutually exclusive subchannels. The opposite-sign dilepton sample targets the bWbW final state and exploits the smallest invariant mass combination of leptons and $b$-jets $\mathrm{M}_{\mathrm{lb}}^{\mathrm{min}}$, in order to suppress the $\mathrm{t} \overline{\mathrm{t}}$ background. A second opposite-sign dilepton channel targets events in which both leptons originate from a $\mathrm{Z}$ boson decay and has further selection criteria based on the increased jet activity. A same-sign dilepton sample accepts events in which at least one $\mathrm{T}$ quark decays to $\mathrm{tZ}$ or $\mathrm{tH}$ and uses control regions to estimate charge misidentification and non-promt lepton backgrounds (Figure 2, left). Finally, a trilepton channel also accepts events in which at least one $\mathrm{T}$ quark decays to $\mathrm{tZ}$ or $\mathrm{tH}$. The nonprompt backgrounds are determined from data, as in the dilepton case. All the subchannels are using observables like $\mathrm{H}_{\mathrm{T}}$ and $\mathrm{S}_{\mathrm{T}}$, in order to exploit the increased jet activity and the invisible particles included. The lower limits for a vector-like T-quark ranges between 687 and $782 \mathrm{GeV}$, depending on the possible branching ratios of the three decay modes.

CMS has also searched for a vector-like heavy B quark having an electric charge $-1 / 3$ [18]. The $\mathrm{B}$ quarks are assumed to be pair produced and decay via $\mathrm{B} \rightarrow \mathrm{tW}, \mathrm{B} \rightarrow \mathrm{bZ}$ or $\mathrm{B} \rightarrow \mathrm{bH}$. Several different mutually exclusive final states are searched in order to be sensitive to the different decay modes, based on the number of selected leptons: single leptons, dileptons with opposite or identical charges, multileptons or all-hadronic without any identified leptons. The first two final states (single lepton and same-sign dilepton) target the tW decay and use as final observable the $\mathrm{S}_{\mathrm{T}}$ variable. The same observable is also used in the multilepton final state which is sensitive to both tW and bZ decay modes. The all-hadronic channel is designed for Higgs bosons decaying to a pair of $b$ quarks. The Higgs boson is identified using CA8 jets that are composed of two b-tagged subjets with an invariant mass constistent with $\mathrm{m}_{\mathrm{H}}$. At least one CA8 jet is required to satisfy these criteria known as Higgs tagging (H-tagging). Finally, in the opposite sign lepton pair channel, the B quark candidate is fully reconstructed from its decay products $\left(\mathrm{B} \rightarrow \mathrm{Zb}, \mathrm{Z} \rightarrow 1 \mathrm{l}\right.$ ), by requiring an $\mathrm{M}_{11}$ close to $\mathrm{Z}$ boson mass and an additional $b$-tagged jet (Figure 2, right). The observed exclusion limit for a vector-like $B$ quark ranges from $740 \mathrm{GeV}$ to $900 \mathrm{GeV}$ depending on the possible branching ratios of the different decay modes.

\section{Search in the 2-dimensional mass plane}

Many BSM models predict final states with two invisible particles [47-49]. The experimental searches are often based on missing energy related observables where the new physics appears in the tail of the distribution. In this case, the establishment of a possible discovery and the model constraining (e.g particle masses) are challenging problems. The suggestion to search in 2-dimensional mass space, for final states with two invisible particles is described in [50]. The reconstruction of mass peaks concentrates signal events in a small region of the two-dimensional mass space whereas background events have no reason to do the same. The reconstructed masses also give valuable information about the particle content and thus the parameters of the model.

CMS has performed a search for anything decaying like top pairs, in their dilepton final state [10]. The analysis is searching simultaneously for both a new heavy top partner $\mathrm{T}^{\prime}$ and a new heavy charged gauge boson $\mathrm{W}^{\prime}$ as predicted by the littlest higgs model $[47,48]$. The selection requires two leptons, two jets and large missing energy with the same energy requirements for all reconstructed objects. The analysis is based on a two-dimensional mass reconstruction of both unknown particles in the $\left[\mathrm{M}_{\mathrm{T}^{\prime}}, \mathrm{M}_{\mathrm{W}^{\prime}}\right]$ plane (Figure 3). The analytic solutions $[36,37]$ together with constraints from the parton distribution functions (PDFs) [51-53] are used to reconstruct the masses of two unknown particles simultaneously. The region of $\mathrm{M}_{\mathrm{T}^{\prime}}$ excluded is in the range $800-920 \mathrm{GeV}$, depending on $\mathrm{M}_{\mathrm{W}^{\prime}}$. 

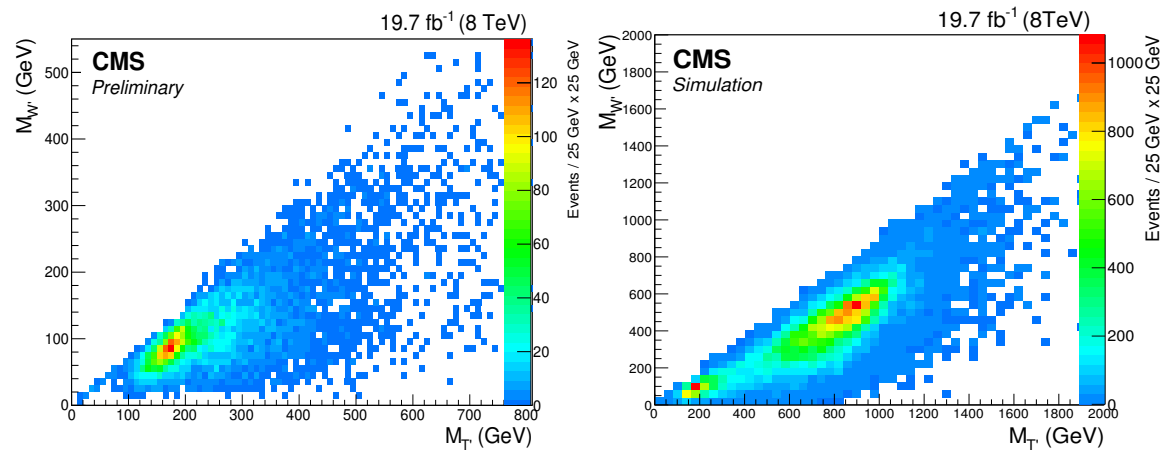

Figure 3. Mass reconstruction in two-dimensional mass space using the 2012 dataset (left) as well as simulated events for the same luminosity of both signal $\left[\mathrm{M}_{\mathrm{T}^{\prime}}=1000 \mathrm{GeV}, \mathrm{M}_{\mathrm{W}^{\prime}}=600 \mathrm{GeV}\right]$ and background processes (right) [10].

\section{Conclusions}

A summary of many CMS searches with final states involving top quarks or heavy top partners is presented. Sophisticed alogorithms are developed for top tagging/mass reconstruction in final states with/without missing energy. No evidence for new physics was found for top quarks associated with possible dark matter particles, resonances decaying to top quarks as well as heavy top-partners. The analysis methods developed in Run1 will facilitate the searches for BSM physics in the new energy regime of Run2.

\section{References}

[1] ATLAS Collaboration, "Observation of a new particle in the search for the Standard Model Higgs boson with the ATLAS detector at the LHC", Phys. Lett. B 716 (2012) 1, arXiv:1207.7214.

[2] CMS Collaboration, "Observation of a new boson at a mass of $125 \mathrm{GeV}$ with the CMS experiment at the LHC”, Phys. Lett. B 716 (2012) 30, arXiv:1207.7235.

[3] CMS Collaboration, "The CMS experiment at the CERN LHC", JINST 3 (2008) S08004.

[4] https://twiki.cern.ch/twiki/bin/view/CMSPublic/PhysicsResultsB2G

[5] CMS Collaboration, "A Cambridge-Aachen (C-A) Based Jet Algorithm For Boosted Top-Jet Tagging”, CMS Physics Analysis Summary CMS-PAS-JME-09-001, 2009.

[6] CMS Collaboration, "Boosted Top Jet Tagging at CMS", CMS Physics Analysis Summary CMSPAS-JME-13-007, 2014.

[7] T. Plehn, M. Spannowsky, M. Takeuchi, and D. Zerwas, "Stop Reconstruction with Tagged Tops", JHEP 10 (2010) 078, arXiv:1006.2833.

[8] CMS Collaboration, "Search for pair production of excited top quarks in the lepton+jet final state", JHEP 06 (2014) 125, arXiv:1311.5357.

[9] CMS Collaboration, "Search for pair production of resonances decaying to a top quark plus a jet in final states with two leptons", CMS Physics Analysis Summary CMS-PAS-B2G-12-008, 2013.

[10] CMS Collaboration, "Search in two-dimensional mass space for $\mathrm{T}^{\prime} \overline{\mathrm{T}}^{\prime} \rightarrow \mathrm{W}^{+\prime} \mathrm{bW}^{-\prime} \overline{\mathrm{b}}$ in the dilepton final state in proton-proton collisions at $\sqrt{s}=8 \mathrm{TeV}$ ", CMS Physics Analysis Summary CMSPAS-B2G-12-025, 2015. 
[11] CMS Collaboration, "Search for Monotop Signatures in proton-proton collisions at 8 Tev", Phys. Rev. Lett. 114 (2015) 101801, hep-ex:1410.1149.

[12] CMS Collaboration, "Search for the production of dark matter in association with top-quark pairs in the single-lepton final state in pp collisions at $\sqrt{s}=8 \mathrm{TeV}$ ", JHEP 06 (2015)121, arXiv:1504.03198.

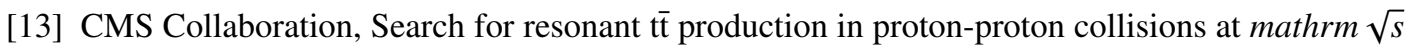
$=8$ TeV", CERN-PH-EP/2015-126, arXiv:1506:03062.

[14] CMS Collaboration, "Search for $\mathrm{W}^{\prime} \rightarrow$ tb in the lepton+jets final state in pp collisions at $\sqrt{s}=$ 8 TeV", JHEP 05 (2014) 108, hep-ex:1402.2176.

[15] CMS Collaboration, "Search for $\mathrm{W}^{\prime} \rightarrow$ tb in the all-hadronic final state", CMS Physics Analysis Summary CMS-PAS-B2G-12-009, 2014.

[16] CMS Collaboration, "Search for Top-Quark Partners with Charge 5/3 in the Same-Sign Dilepton Final State", Phys. Rev. Lett. 112 (2014) 171801, hep-ex:1212:2391.

[17] CMS Collaboration, "Inclusive search for a vector-like T quark with charge $2 / 3$ in pp collisions at $\sqrt{s}=8$ TeV", Phys. Lett. B 729 (2014) 149, hep-ex:1311.7667.

[18] CMS Collaboration, "Search for pair-produced vector-like B quarks in proton-proton collisions at $\sqrt{s}=8 \mathrm{TeV}$ ", CMS Physics Analysis Summary CMS-PAS-B2G-13-006, 2015, hepex:1507.07129.

[19] ATLAS Collaboration, "Search for dark matter candidates and large extra dimensions in events with a jet and missing transverse momentum with the ATLAS detector", JHEP 04 (2013) 075, arXiv: 1210.4491.

[20] CMS Collaboration, "Search for dark matter, extra dimensions, and unparticles in monojet events in proton-proton collisions at $\sqrt{s}=8 \mathrm{TeV}$ ", (2014), arXiv:1408.3583.

[21] ATLAS Collaboration, "Search for dark matter candidates and large extra dimensions in events with a photon and missing transverse momentum in pp collision data at $\sqrt{s}=7 \mathrm{TeV}$ with the ATLAS detector", Phys. Rev. Lett. 110, (2013), 011802, arXiv:1209.4625.

[22] CMS Collaboration, "Search for Dark Matter and Large Extra Dimensions in pp Collisions Yielding a Photon and Missing Transverse Energy", Phys. Rev. Lett. 108, (2012), 261803, arXiv:1204.0821.

[23] J. Andrea, B. Fuks, and F. Maltoni, "Monotops at the LHC", Phys. Rev. D 84 (2011) 074025 , arXiv:1106.6199.

[24] J.-L. Agram et al., "Monotop phenomenology at the Large Hadron Collider", Phys. Rev. D 89 (2014) 014028, arXiv:1311.6478.

[25] M. Beltrán, D. Hooper, E.W. Kolb, Z.A.C. Krusberg and T.M.P. Tait, Maverick dark matter at colliders, JHEP 09 (2010) 037, arXiv:1002.4137.

[26] K. Cheung, K. Mawatari, E. Senaha, P.-Y. Tseng and T.-C. Yuan, The top window for dark matter, JHEP 10 (2010) 081, arXiv:1009.0618.

[27] T. Lin, E.W. Kolb and L.-T. Wang, Probing dark matter couplings to top and bottom quarks at the LHC, Phys. Rev. D 88 (2013) 063510, arXiv:1303.6638.

[28] Y. Bai, H.-C. Cheng, J. Gallicchio and J. Gu, "Stop the top background of the stop search", JHEP 07 (2012) 110, arXiv:1203.4813.

[29] R. M. Harris and S. Jain, "Cross sections for leptophobic topcolor Z' decaying to top-antitop", Eur. Phys. J. C 72 (2012) 2072, arXiv:1112.4928.

[30] K. R. Lynch, S. Mrenna, M. Narain, and E. H. Simmons, "Finding Z' bosons coupled preferentially to the third family at CERN LEP and the Fermilab Tevatron", Phys. Rev. D 63 (2001) 035006, arXiv:hep-ph/0007286. 
[31] K. Agashe et al., "LHC signals from warped extra dimensions”, Phys. Rev. D 77 (2008) 015003, arXiv:hep-ph/0612015.

[32] H. Georgi, L. Kaplan, D. Morin and A. Schenk, "Effects of top compositeness", Phys. Rev. D 51 (1995) 3888, hep-ph/9410307.

[33] B. Hassanain, J. March-Russell and J.G. Rosa, "On the possibility of light string resonances at the LHC and Tevatron from Randall-Sundrum throats", JHEP 07 (2009) 077, arXiv:0904.4108.

[34] C. Burges and H. J. Schnitzer, "Virtual Effects of Excited Quarks as Probes of a Possible New Hadronic Mass Scale", Nucl. Phys. B 228 (1983) 464.

[35] C. Csaki, Y. Grossman, and B. Heidenreich, "MFV SUSY: A Natural Theory for R-Parity Violation", Phys. Rev. D 85 (2012) 095009, arXiv:1111.1239.

[36] L. Sonnenschein, “Analytical solution of tt̃ dilepton equations”, Phys. Rev. D 73 (2006) 054015, arXiv:hep-ph/0603011.

[37] L. Sonnenschein, "Algebraic approach to solve t anti-t dilepton equations", Phys. Rev. D 72, 095020, 2005, arXiv:hep-ph/0510100.

[38] M. Schmaltz, "Little Higgs review", Ann. Rev. Nucl. Part. Sci. 55 (2005) 229, hep-ph/0502182.

[39] T. Appelquist, H.-C. Cheng and B.A. Dobrescu, "Bounds on universal extra dimensions", Phys. Rev. D 64 (2001) 035002, hep-ph/0012100.

[40] CMS collaboration, "Search for new physics in final states with a lepton and missing transverse energy in pp collisions at the LHC, Phys. Rev. D 87 (2013) 072005, arXiv:1302.2812.

[41] CMS collaboration, "Search for exotic resonances decaying into WZ/ZZ in pp collisions at $\sqrt{s}$ =7 TeV, JHEP 02 (2013) 036, arXiv:1211.5779.

[42] CMS collaboration, "Search for narrow resonances using the dijet mass spectrum in pp collisions at $\sqrt{s}=8$ TeV", Phys. Rev. D 87 (2013) 114015, arXiv:1302.4794.

[43] M. Nemevšek, F. Nesti, G. Senjanović and Y. Zhang, "First Limits on Left-Right Symmetry Scale from LHC Data", Phys. Rev. D 83 (2011) 115014, arXiv:1103.1627.

[44] CMS Collaboration, "Identifying Hadronically Decaying Vector Bosons Merged into a Single Jet”, CMS Physics Analysis Summary CMS-PAS-JME-13-006, 2013.

[45] J. A. Aguilar-Saavedra, R. Benbrik, S. Heinemeyer, and M. Perez-Victoria, "Handbook of vectorlike quarks: Mixing and single production", Phys. Rev. D 88 (2013) 094010.

[46] A. Djouadi and A. Lenz, "Sealing the fate of a fourth generation of fermions", Phys. Lett. B 715 (2012) 310.

[47] T. Han, H. E. Logan, B. McElrath, and L.-T. Wang, "Phenomenology of the little Higgs model", Phys. Rev. D 67 (2003) 095004, arXiv:hep-ph/0301040.

[48] Jürgen Reuter, Marco Tonini, Maikel de Vries, "Littlest Higgs with T-parity: Status and Prospects", JHEP 1402 (2014) 053, arXiv:1310.2918.

[49] S. P. Martin, “A Supersymmetry primer”, Adv. Ser. Direct. High Energy Phys. 18 (1997) 1, arXiv:hep-ph/9709356.

[50] G. Anagnostou, "Model Independent Search in 2-Dimensional Mass Space”, in 2nd International Conference on New Frontiers in Physics, EPJ Web of Conferences, Volume 71, 2014.

[51] R. H. Dalitz and G. R. Goldstein, "The decay and polarization properties of the top quark, Phys. Rev. D 45 (1992).

[52] D0 Collaboration, "Measurement of the top quark mass in final states with two leptons", Phys. Rev. D 80, 092006 (2009).

[53] CMS Collaboration, "Measurement of the top quark mass using proton-proton data at $\sqrt{s}=7$ and 8 TeV", CERN-PH-EP-2015-234, arXiv:1509.04044. 